INRA Prod. Anim., 2002, 15 (3), 185-198
P. ORGEUR ${ }^{1}$, J. LE DIVIDICH ${ }^{2}$, V. COLSON ${ }^{1}$, M.C. MEUNIER-SALAÜN ${ }^{2}$

${ }^{1}$ INRA, UMR Physiologie de la Reproduction et des Comportements, 37380 Nouzilly

${ }^{2}$ INRA, UMR Veau et Porc, 35590 Saint-Gilles

Courriel : orgeur@tours.inra.fr

\section{La relation} mère-jeune chez les porcins : de la naissance au sevrage

Une nouvelle directive européenne, applicable en 2003, révise les normes minimales relatives à la protection des porcs, notamment l'âge minimal à partir duquel les porcelets peuvent être sevrés. En effet, le sevrage représente une situation de stress majeur pour le porcelet : en plus du changement de logement et d'alimentation qu'il implique, il correspond à la privation de la mère et le plus souvent à un changement de groupe social. Cet article fait le point sur l'établissement du lien mère-jeune, son évolution et l'impact des pratiques d'élevage notamment en matière de bien-être.

\section{Résumé}

L'élevage intensif du porc impose des contraintes qui peuvent être néfastes à son bien-être. Chez le sanglier ou le porc domestique en conditions semi-naturelles, la femelle parturiente vit en groupe matriarcal et confectionne un nid destiné à dissimuler et protéger les jeunes. Un lien s'établit entre la truie et ses porcelets et la période d'allaitement dure au minimum 10 à 12 semaines. Les conduites d'élevage actuelles ont fondamentalement modifié le mode de vie du porc. Les truies sont généralement placées en cases individuelles et ne peuvent confectionner un nid. Pendant toute la durée de l'allaitement, les contacts avec les porcelets sont réduits au minimum et initiés par ces derniers. Il existe néanmoins des échanges vocaux et olfactifs facilitant la localisation des tétines et conduisant à une reconnaissance réciproque mèrejeunes, effective dans les 48 heures. Labsorption rapide de colostrum est essentielle pour la protection immunitaire et l'apport énergétique. Le rythme des allaitements est d'environ 1 par heure pendant les premières semaines de lactation, avant de diminuer progressivement. Les porcelets développent rapidement une grande fidélité aux tétines, à l'issue de combats souvent intenses. Le lien mère-jeunes n'est pas aussi sélectif que chez les ruminants et des adoptions sont facilement réalisables lorsque les porcelets sont âgés de moins de trois jours. Ultérieurement, l'adoption de quelques porcelets devient difficile et ceux-ci tardent à s'engager à la tétée. Cependant, la substitution d'une portée entière est facilement acceptée par la truie, même après 3 semaines d'allaitement. Le sevrage, interdit en deçà de 4 semaines sauf dans les élevages en bandes, est réalisé à cet âge dans la majorité des élevages intensifs. Il peut être pratiqué dès que les porcelets sont âgés d'une semaine, mais uniquement si leur survie est en jeu. Les perturbations comportementales et zootechniques sont d'autant plus négatives que le sevrage est précoce. La prise en compte du bien-être de l'animal impose des précautions visant à ne pas placer l'animal dans une situation de peur ou d'anxiété et à lui permettre d'exprimer les comportements dits normaux de l'espèce.
L'amélioration des techniques d'élevage et la sélection des truies sur la prolificité ont permis d'augmenter fortement leur productivité. Ainsi, le nombre de porcelets sevrés par truie et par an est passé de 20,2 en 1980 à 25,2 en 2000 (ITP 2001). Cependant, ces conduites modernes ont généré des contraintes pour l'animal, ainsi que des conséquences parfois néfastes pour l'environnement qu'il est désormais indispensable de prendre en compte. Ces orientations nouvelles de l'élevage ont déjà fait l'objet de réglementations strictes en matière de logement et de conduite des animaux. Il est probable que leur évolution tiendra de plus en plus compte du bien-être de ces animaux. A ce titre, la directive communautaire 91/630/CE, adoptée en novembre 1991 et transcrite par un arrêté français datant de janvier 1994, concerne les normes minimales relatives à la protection des porcs et notamment à leur logement. Cette directive stipule également que les porcelets ne doivent pas être séparés de leur mère avant l'âge de 21 jours, sauf si la non séparation est préjudiciable au bien-être ou à la santé de la truie et de ses porcelets.

En juin 2001, la directive européenne 91/630 a été révisée, donnant lieu à de nouveaux textes sur les dispositions générales (2001/88/CE) et relatifs aux annexes (2001/93/CE). Ainsi, l'âge limite du sevrage est désormais fixé à 28 jours. Toutefois, un sevra- 
ge à 21 jours reste possible dans les conditions de conduite en bandes ${ }^{(1)}$. Cette décision est applicable à partir du 1er janvier 2003.

En matière de bien-être du porc, les directives européennes prennent en compte la possibilité pour l'animal d'exprimer les comportements dits normaux de l'espèce. L'expression de ces comportements paraît d'autant plus valide que l'animal est placé dans des conditions naturelles. Dans ce cadre, le sanglier a fait l'objet d'études contribuant à l'évaluation des conséquences de modifications imposées au porc par l'éleveur au fil de la modernisation de l'élevage. En élevage intensif français, les truies allaitantes sont dans la majorité des cas bloquées en cases individuelles de mise bas, sur un sol en caillebotis, pendant une période de 4 à 5 semaines.

L'objectif de cet article est d'examiner dans les conditions les plus représentatives de l'élevage français, l'implication des pratiques d'entretien des animaux pendant la phase d'allaitement sur les relations entre la truie et ses porcelets. Nous nous attacherons à la description des différentes étapes de la relation mère-jeunes allant de la naissance au sevrage. Les conséquences de l'application des pratiques d'élevage en matière de bien-être animal seront également évoquées.

\section{1 / La mise bas}

Chez le sanglier, la structure sociale repose sur l'existence d'un groupe matriarcal au sein duquel il existe une synchronisation de la reproduction et donc des naissances. Les laies quittent le groupe social et construisent un nid à l'approche de la mise bas. Celui-ci a pour fonction de dissimuler jeunes et mère et d'assurer une protection des marcassins contre le froid auquel ils sont sensibles au début de leur vie (Jones 1966).

Le comportement des porcs domestiques présente potentiellement de grandes similitudes avec celui des sangliers. La truie, lorsqu'elle en a la possibilité, confectionne également un nid dans les heures qui précèdent la parturition (Jensen 1988). C'est le cas en conditions semi-naturelles : la truie, comme la laie, utilise les matériaux auxquels elle a accès. Dans le cas de l'élevage en plein air (moins de $10 \%$ des élevages français), les truies disposent dans leur case, de paille pour aménager ce nid.
Dans les conditions actuelles d'élevage et dans la très grande majorité des cas, les truies n'ont plus la possibilité de confectionner un nid avant de mettre bas. Elles sont placées, environ une semaine avant la parturition, en cases individuelles dépourvues de tout substrat. La case est pourvue d'un système de blocage de la truie, ce qui limite les pertes de porcelets par écrasement, mais réduit aussi les possibilités d'interactions avec ces derniers, dans la mesure où eux seuls peuvent initier le contact avec la mère.

A la naissance, le porcelet pèse environ deux cents fois moins que sa mère. Ses besoins vitaux en colostrum l'incitent à rechercher le contact avec celle-ci et le rendent physiquement vulnérable. L'écrasement par la truie peut dépasser $15 \%$ de mortalité dans les trois premiers jours post partum (English et Morrison 1984, Spicer et al 1986, Svendsen et al 1986). Les cases de maternité sont équipées d'un système de chauffage localisé indispensable au maintien de l'homéostasie thermique du nouveau-né. En raison de leur mauvaise isolation thermique, l'optimum thermique des nouveaunés est très élevé, de l'ordre de $32-34^{\circ} \mathrm{C}$ dans les premières heures qui suivent la naissance. Il s'abaisse à $26-28^{\circ} \mathrm{C}$ dès que leur comportement de groupe se manifeste (Herpin et Le Dividich 1995). Malgré tout, un compromis doit cependant être trouvé afin que la température du local convienne à la fois aux besoins du porcelet et à ceux de la truie. Cette source de chaleur, éloignée de la truie, généralement localisée dans un coin de la case, maintient les petits dans une zone de sécurité permettant de limiter les risques d'écrasement (Weary et al 1996).

Lors de la mise bas, la truie est en général couchée sur le flanc. Les porcelets se libèrent seuls des enveloppes foetales et commencent rapidement à explorer leur environnement à la recherche de la mamelle (Jones 1966, Randall 1972). Chez la truie, la durée de la mise bas est très variable, de 60 à plus de 360 min, en partie selon la taille de la portée. Une durée d'expulsion longue (supérieure à 4 heures) entraîne une augmentation de la mortinatalité par anoxie (Léon et Madec 1992). L'anoxie n'est pas toujours immédiatement fatale. Toutefois, elle retarde et réduit la prise de colostrum des survivants (cf. 2.2.), perturbant dans un premier temps la fonction de thermorégulation, ce qui conduit à diminuer le taux de survie ultérieure des porcelets (Herpin et al 1996 ; tableau 1).

Tableau 1. Mortalité post-natale (d'après Herpin et al 1996).

\begin{tabular}{|l|c|c|c|}
\hline & $\begin{array}{c}\text { Porcelets morts } \\
\text { avant } 10 \text { jours }\end{array}$ & $\begin{array}{c}\text { Porcelets vivants } \\
\text { à } 10 \text { jours }\end{array}$ & Valeur de P \\
\hline Poids à la naissance $(\mathrm{kg})$ & 1,064 & 1,342 & 0,01 \\
Effectif & 25 & 82 & 0,01 \\
\hline Délai naissance-1er contact mamelle $(\mathrm{min})$ & 55 & 23 & \\
Effectif & 24 & 67 & 0,01 \\
\hline Température rectale à $24 \mathrm{~h}\left({ }^{\circ} \mathrm{C}\right)$ & 36,5 & 38,6 & \\
Effectif & 18 & 84 & \\
\hline
\end{tabular}

(1) La conduite en bandes consiste à synchroniser $\mathrm{N}$ truies pour la reproduction. Une rotation sur 3 semaines peut se faire: la première semaine, une bande (B) met bas, la deuxième semaine, les porcelets de la bande (B-1) sont sevrés, la troisième semaine, les truies de cette bande (B-1) sont ré inséminées. 


\section{2 / La lactation}

\section{1 / La recherche de la mamelle}

Le porcelet est suffisamment mature sur le plan physique à la naissance pour se mouvoir avec autonomie. Aussitôt né, il commence à explorer le corps de sa mère à la recherche de la mamelle. Des stimulations multi-sensorielles l'aident à la localiser. Les informations olfactives semblent jouer un rôle essentiel. En effet, dès la naissance, le porcelet est attiré par des odeurs émanant du corps maternel telles que celles des liquides foetaux et du lait (Rohde-Parfet et Gonyou 1991) et des indices olfactifs émis au niveau de la mamelle favorisent la saisie de la tétine (Morrow-Tesch et McGlone 1990). L'attraction du porcelet nouveau-né peut également être influencée par d'autres stimulations. Selon Rohde-Parfet et Gonyou (1991), les porcelets mâles sont, par exemple et de manière inexpliquée, plus sensibles que les femelles aux vocalisations de la truie parturiente. Les nouveau-nés montrent aussi une préférence tactile pour une surface douce telle que celle de la mamelle (Welch et Baxter 1986). La température de la région abdominale et l'orientation des soies de la truie auraient également un rôle facilitateur dans la recherche active de la mamelle. Une combinaison de ces différents stimuli potentiels facilite la localisation et la saisie initiales des tétines. En moyenne, les porcelets localisent les tétines entre 10 à 35 minutes après la naissance. Les porcelets les plus gros sont plus performants dans leur recherche que ceux de poids plus faible (Jones 1966).

\section{2 / La prise colostrale}

Le colostrum, sécrétion initiale de la mamelle, a trois rôles essentiels : nutritionnel (apport de nutriments pour le maintien de l'homéothermie et de la glycémie), immunitaire (transfert d'immunoglobulines qui ne peut se faire en fin de gestation du fait de la placentation épithélio-choriale imperméable, transfert de lymphocytes) et physiologique (apport de facteurs de croissance).

A la naissance, le porcelet est dépourvu de toute protection immunitaire et ses réserves énergétiques sont très faibles (12 fois plus faibles que chez l'enfant) (Herpin et Le Dividich 1995). Dans ces conditions, une consommation rapide et importante de colostrum est indispensable à sa survie. Habituellement, la première prise colostrale a lieu dans les 20-30 minutes qui suivent la naissance, mais elle peut être retardée jusqu'à 60 min et davantage si le porcelet est léger (Hoy et al 1994) ou fortement anoxié à la naissance (Herpin et al 1996). La quantité moyenne de colostrum consommé par les porcelets est de l'ordre de 300-340 g au cours du premier jour de vie (Le Dividich et Noblet 1981, Milon et al 1983), mais il peut en ingérer jusqu'à 600 g (Le Dividich et al 1997). Toutefois, l'évolution du poids le premier jour (de -136 à +230 g, Thompson et Fraser 1988) indique que la consommation de colostrum est très variable. Le froid (Le Dividich et Noblet 1981), la pré- maturité (Milon et al 1983) et l'hypoxie ou anoxie néonatale ont des effets néfastes sur cette consommation (Herpin et al 1996). Les porcelets les plus légers et ceux qui naissent en dernier sont les moins favorisés. Les plus légers car ils sont moins vigoureux et les derniers nés car le colostrum est déjà moins riche en immunoglobulines, tandis que dès la fin de la mise bas, la mamelle n'est plus entièrement disponible. Une consommation insuffisante et tardive de colostrum est une cause essentielle de mortalité précoce en raison du déficit énergétique conduisant à une hypothermie fatale du porcelet. Selon Blecha et Kelley (1981) et Hendrix et al (1978) les porcelets qui meurent plus tardivement au cours de la phase d'allaitement présentent une immunité humorale insuffisante. Dès 14-24 h d'âge, les taux d'IgG sériques sont de 30 à $40 \%$ plus faibles que chez les survivants. Dans la mesure où la proportion de porcelets de faible poids (Le Dividich 1999) et la durée de mise bas augmentent dans les portées de grande taille, on peut supposer une prise colostrale insuffisante chez un plus grand nombre de porcelets dans ces portées. Malheureusement, on connaît encore mal les facteurs qui contrôlent la production colostrale chez la truie.

Récemment, une relation positive a été mise en évidence entre les taux sériques d'IgG à $4-7$ jours et à 28 jours d'âge (la veille du sevrage). En d'autres termes plus l'immunité colostrale est élevée, plus l'immunité active développée par les porcelets est elle-même élevée au moment du sevrage (Rooke et Bland 2002). L'immunité colostrale pourraitelle avoir des répercussions sur la santé du porcelet au sevrage ? C'est une question importante à laquelle il n'existe pas encore de réponse.

Parmi les différentes classes d'immunoglobulines présentes dans le colostrum, les IgG sont responsables de la neutralisation des virus et des toxines et stimulent la phagocytose. Pendant les 6 premières heures post partum, elles représentent la quasi-totalité des protéines présentes. Puis leur quantité diminue progressivement, pour être minimale après 2 à 3 semaines, période où les porcelets sont particulièrement vulnérables.

Les IgA protègent les muqueuses contre les agressions microbiennes. Leur concentration, peu importante dans les premiers jours, s'accroît après 2 semaines pour atteindre environ $40 \%$ de la totalité des matières protéiques du lait en fin de lactation (Klobasa et al 1987). Elles assurent, jusqu'au sevrage, l'immunité locale passive de l'intestin.

Les IgM contribuent essentiellement à l'élimination des agents infectieux et sont à l'origine des réactions immunitaires humorales. Leur transfert vers le jeune se fait dans les 36 heures après la parturition. Elles confèrent une protection efficace, tout en provoquant un blocage de l'immunisation active (Salmon et al 1990). Celle-ci s'installe après environ 3 semaines, grâce aux stimulations antigéniques de l'environnement dont l'aliment soli- 
de ingéré par le porcelet. Ainsi quand une alimentation solide est proposée plus précocement à des porcelets, à l'âge de 6-7 jours par exemple, leur immunité locale active se met en place plus tôt (Orgeur et al 2001).

\section{3 / Le déroulement de la tétée}

\section{a / Le réflexe d'éjection du lait}

La truie requiert une stimulation importante pour que le réflexe d'éjection du lait apparaisse. Toutefois, pendant la phase colostrale, ce mécanisme ne semble pas nécessaire et le colostrum est disponible en continu. Le réflexe d'éjection de lait comprend une voie nerveuse ascendante et une voie humorale descendante. Le point de départ de l'arc réflexe se situe au niveau des récepteurs sensoriels du mamelon et le point d'arrivée est constitué par les neurones ocytocinergiques du système hypothalamo-neurohypophysaire. Locytocine libérée gagne la glande mammaire par voie sanguine et provoque la contraction des cellules myoépithéliales entraînant l'expulsion du lait des acini (Ely et Petersen 1941).

\section{b / La séquence d'allaitement}

Une séquence d'allaitement dure entre 2 et 6 minutes pendant lesquelles les partenaires (mère et jeunes) échangent des informations de nature essentiellement auditives et tactiles (Fraser 1980, Ellendorf et Poulain 1984). Les informations olfactives et gustatives fournies au porcelet par le contact avec la mamelle et l'ingestion du lait ont sans doute elles aussi un rôle important dans la reconnaissance de la mère par les jeunes, comme cela a été montré dans d'autres espèces. L'ensemble d'une séquence peut être décomposé en 6 étapes (tableau 2). Celles-ci vont de la mise en position de la truie (couchée sur le flanc) et du rassemblement des porcelets à la mamelle, au changement de position de la truie et des porcelets marquant la fin de l'allaitement, en passant par la libération d'ocytocine et par l'éjection du lait (25 à 35 secondes) pendant laquelle les jeunes sont totalement immobiles (Algers et Jensen 1991). L'allaitement se caractérise aussi par la succession de séquences de grognements de la truie et de massages-succions des porcelets, variant en fréquence et en intensité.

\section{4 / Evolution du rythme de tétées au cours de la lactation}

Passée la phase colostrale, les allaitements se déroulent à un rythme régulier d'environ un par heure dans la journée et deux par heure la nuit au début de la lactation. Au début de la phase d'allaitement, le rythme des tétées est le même chez la laie et chez la truie, quel que soit son mode d'élevage. L'intervalle moyen entre tétées varie de 45 à 50 minutes (Ellendorf et al 1982, Auldist et King 1995, Arey et Sancha 1996). Ce rythme diminue au cours de la lactation. Selon Schouten (1986) les porcelets passent $30 \%$ de leur temps en "activité tétée" au cours des deux premières semaines de lactation, et seulement $15 \%$ à la sixième semaine. Dans un système d'élevage où la truie est libre dans sa case de maternité, ce qui lui permet de refuser d'allaiter plus facilement qu'en contention, la fréquence quotidienne de tétées peut même se réduire à une moyenne de 4,8 par jour après 28 jours de lactation (Boe 1993). D'une manière générale, les conditions de logement des animaux peuvent moduler assez largement le rythme d'allaitement. Ainsi des truies libres allaitent moins fréquemment que des truies entravées, les porcelets de ces dernières sont plus lourds au sevrage (Pajor et al 1997). Le logement collectif des truies allaitantes semble par ailleurs accélérer le processus de sevrage spontané. Les truies évitent davantage les porcelets et ces derniers sont engagés dans une activité sociale plus importante avec leurs congénères (Stolba et al 1990, Blackshaw et al 1994, Pedersen et al 1998).

L'initiative de l'allaitement revient à la mère, particulièrement pendant les premiers jours ; par la suite, elle peut revenir également aux jeunes (Fraser 1980, Martineau 1998). Les allaitements sont le plus souvent synchronisés entre portées situées dans une même maternité et il en est de même chez des laies appartenant au même groupe social. Au sein de chaque portée, les tétées sont également très synchronisées, tous les individus tétant en même temps (de Passillé et Rushen 1989).

La phase de massages avant tétée (prététée) joue un rôle primordial pour la libération de l'ocytocine qui conditionne l'excrétion du lait. La phase finale de massages (posttétée) a également un rôle important pour la

Tableau 2. Déroulement des séquences d'allaitement chez le porc (d'après Fraser 1980).

\begin{tabular}{|l|l|l|}
\hline & \multicolumn{1}{|c|}{ Truie } & \multicolumn{1}{c|}{ Porcelets } \\
\hline Etape 1 - $30 \mathrm{sec}$ & $\begin{array}{l}\text { Position d'allaitement } \\
\text { Grognements d'appel }\end{array}$ & $\begin{array}{l}\text { Rassemblement aux mamelles } \\
\text { Massage initial (prémassage) }\end{array}$ \\
\hline Etape 2 - $60 \mathrm{sec}$ & $\begin{array}{l}\text { Grognements rythmiques } \\
\text { (augmentation de la fréquence) }\end{array}$ & $\begin{array}{l}\text { Compétition aux mamelles } \\
\text { Massages rythmiques }\end{array}$ \\
\hline Etape 3 - 20 à 30 sec & $\begin{array}{l}\text { Augmentation rapide de la } \\
\text { fréquence des grognements } \\
\text { - Décharge d'ocytocine }\end{array}$ & Succions lentes (1-2/sec) \\
\hline Etape 4 - 20 sec & Début d'éjection du lait & Succions rapides (4-5/sec) \\
\hline Etape 5 - quelques sec ou min & $\begin{array}{l}\text { Diminution de la fréquence des } \\
\text { grognements (arrêt de l'éjection du lait) }\end{array}$ & $\begin{array}{l}\text { Succions lentes ou } \\
\text { massage (postmassage) }\end{array}$ \\
\hline Etape 6 & Changement de position - repos & $\begin{array}{l}\text { Repos, postmassage ou éloignement } \\
\text { de la mamelle }\end{array}$ \\
\hline
\end{tabular}


sécrétion de prolactine et donc la synthèse du lait. Selon Jensen (1988), le temps moyen passé par les porcelets à masser la mamelle de leur mère (truies primipares en liberté) avant chaque tétée est stable pendant les 10 premières semaines de lactation, puis il augmente significativement entre la dixième et la quinzième. Le temps moyen de massage posttétée diminue significativement entre la première et la quatrième semaine, puis reste stable (figure 1). Dans la première semaine, cette activité post-tétée varie de 30 à 100 minutes par jour (Jensen et Recen 1989).

Les quantités de lait consommées par le porcelet sont, en valeur absolue, maximales entre la $2^{\text {ème }}$ et la $4{ }^{\text {ème }}$ semaine de lactation et varient entre 700 et $900 \mathrm{~g} / \mathrm{j}$ (SalmonLegagneur et Aumaître 1962, Noblet et Etienne 1987, Beyer et Jentch 1994). Mais, exprimées en $\mathrm{g} / \mathrm{kg}$ de poids corporel, elles sont maximales au cours de la $1^{\text {ère }}$ semaine et représentent en moyenne $250 \mathrm{~g} / \mathrm{kg}$. Elles décroissent par la suite à environ 120-140 g/kg au cours de la $4^{\text {ème }}$ semaine de lactation.

Lintensité des massages exercés par les porcelets module la production laitière qui est également très dépendante de l'alimentation et de l'aptitude laitière de la truie (Algers et Jensen 1991). Les porcelets ne réduisent cependant pas leurs efforts de massage, en réponse à une augmentation de la quantité de lait produit (Jensen et al 1998). La quantité

Figure 1. Evolution des massages pré et posttétée au cours de la lactation de la truie en liberté (d'après Jensen 1988).

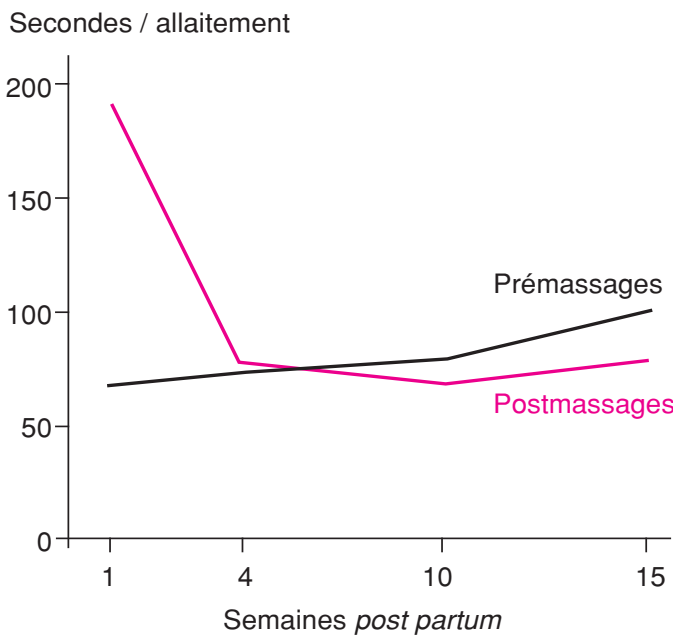

moyenne de lait tétée par une portée varie de 2-3 kg environ le premier jour (J. Le Dividich, communication personnelle) à 11-13 kg vers 21 jours de lactation (Etienne et al 1998). La courbe de lactation d'une truie passe par un maximum vers la troisième ou quatrième semaine, avec une grande variation selon les truies. Le tarissement naturel a lieu vers la fin du deuxième mois et est lié à une diminution de la stimulation, mais l'allaitement peut durer jusqu'à 17 semaines en conditions seminaturelles (Jensen et Recen 1989).

\section{5 / Ordre de tétée et fidélité aux tétines}

Chez la laie, les tétines les plus postérieures sont considérées potentiellement comme les plus productrices (Delcroix 1989), alors que chez la truie, ce sont les plus antérieures qui produisent le plus de lait (Dantzer et al 1986). Les porcelets les plus vifs à la naissance s'y fixent de préférence et leurs massages plus vigoureux contribuent à les rendre plus performantes (Buchenauer et Dannenmann 1979). Peu après la naissance, les porcelets choisissent une tétine ou une paire de tétines qu'ils tendent ensuite à conserver tout au long de la période d'allaitement. L'ordre de tétée se développe progressivement dans les jours suivant la naissance. La recherche, puis la fidélité à une tétine ou à une paire de tétines donnent lieu à des combats entre porcelets, d'autant plus acharnés que le nombre de porcelets est élevé par rapport au nombre de tétines (Hartsock et al 1977). Les interactions agressives sont principalement orientées vers les tétines antérieures, les plus convoitées. Ces combats peuvent générer des blessures, tant des porcelets eux-mêmes que de la truie, au niveau de la mamelle. C'est l'une des raisons du coupage ou du meulage des dents à la naissance. Le poids à la naissance et la vigueur qui y est généralement associée sont des éléments déterminants pour la probabilité de succès dans les combats et la possibilité d'accès aux tétines antérieures. Une hiérarchie s'établit alors et en général les porcelets les plus petits sont amenés à utiliser les tétines postérieures (Scheel et al 1977 ; figure 2). Il existe même, selon de Passillé et al (1988), une catégorie de porcelets considérés comme atypiques qui ne réussissent pas à s'attribuer une tétine. Leur rythme de tétée est plus faible et ils continuent à se battre pour l'accès à la mamelle tout au long de la

Figure 2. Accès à la mamelle (d'après Scheel et al 1977).

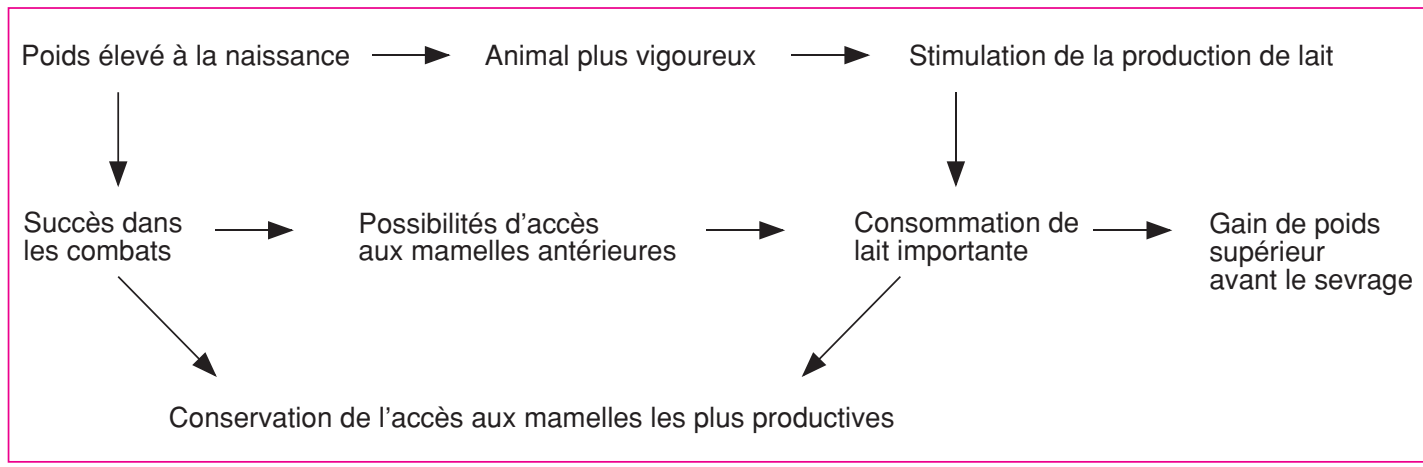


période d'allaitement, accusant un retard de développement considérable. A l'opposé les porcelets vigoureux tirent un meilleur parti de leur position. Une forte corrélation a en effet été mise en évidence entre la position à la mamelle et la croissance (Fraser 1973, Hartsock et al 1977, Rossillon-Warnier et Paquay 1984). Au sevrage, les porcelets utilisant les mamelles antérieures ont un poids supérieur, ce qui facilite leur accès à un statut social de type dominant dans les groupes sociaux formés à l'issue du sevrage.

$\mathrm{Au}$ bout de 24 heures, $50 \%$ des tétées ont lieu sur la paire de tétines préférée. Après 3 jours, ce pourcentage atteint $86 \%$ et au bout de 10 jours, la fidélité est quasi-parfaite (de Passillé et al 1988). Cette mise en place peut cependant être perturbée dans les portées de grande taille, lorsque le nombre de porcelets est supérieur au nombre de tétines fonctionnelles. Dans des portées de 14 porcelets ou plus, seulement $80 \%$ des porcelets tètent sur une seule paire de tétines au bout de 13 jours (Saez 2000).

\section{6 / La reconnaissance mère- jeunes}

La mise en place d'un lien entre la mère et ses jeunes est une étape importante pour la survie du nouveau-né. Ce lien correspond à une relation privilégiée entre la mère et les jeunes de la portée. Il s'exprime par une reconnaissance mutuelle et par des conduites d'évitement ou d'agressivité de la mère vis-àvis de jeunes étrangers.

L'apparition du comportement maternel semble être le résultat d'interactions complexes entre les signaux provenant des porcelets (olfactifs, visuels, sonores, tactiles) et les mécanismes internes de régulation hormonale de la truie (œestrogènes, ocytocine, prolactine) au cours de la période périnatale et durant la lactation (Deprès et Caritez 1991). Le lien entre la truie et les porcelets est toutefois moins sélectif que chez la plupart des ongulés. En effet, la truie accepte facilement d'allaiter des porcelets étrangers, notamment dans les premières heures qui suivent la mise bas (Deprès et Caritez 1991). Lorsque plusieurs truies sont regroupées avec leur portée, l'allaitement de porcelets étrangers est en effet fréquemment observé. C'est également le cas chez le sanglier. Un lien préférentiel est cependant établi avec les jeunes de la portée qui restent auprès de leur mère au sein du groupe et réagissent principalement à ses grognements d'appel (Delcroix 1989).

En dehors des périodes de tétées, les jeunes passent $90 \%$ de leur temps près de la truie durant les trois premiers jours de vie. Dans cette période initiale, les porcelets approchent leur mère quelle que soit sa posture, d'où des risques importants d'écrasement. Par ailleurs, le développement du lien serait initié par la mère dès le premier jour (Blackshaw et Hagels 1990), mais les jeunes y prennent une part active dès le jour de leur naissance et participent à la consolidation de ce lien les jours suivants.
Figure 3. Discrimination des porcelets par la truie (d'après Horrell et Hodgson 1992).

Temps (s) d'attention dirigée vers les porcelets

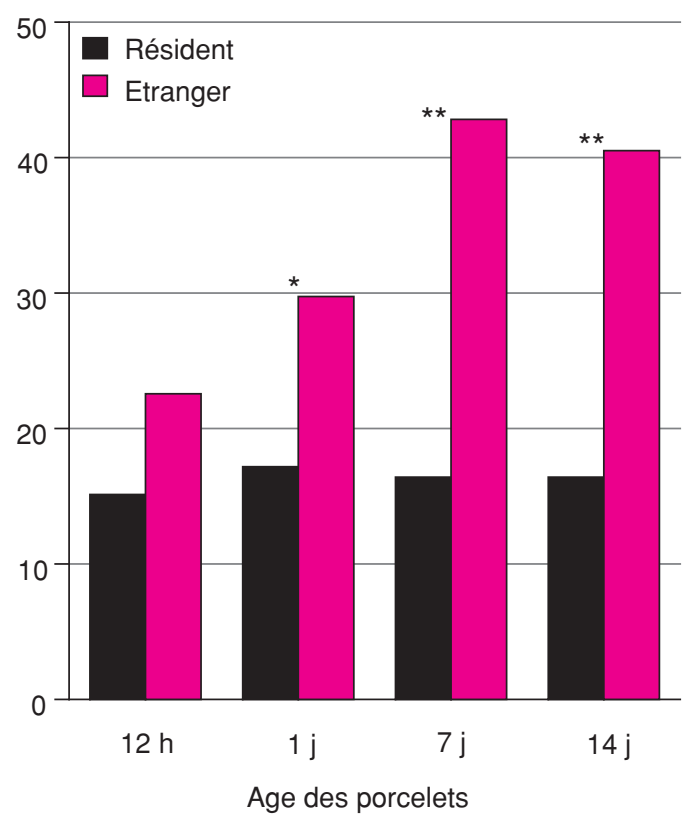

Dès 24 à 36 heures post-partum, la truie est capable de reconnaître ses propres jeunes (Horrell et Hodgson 1992 ; figure 3). Les informations olfactives semblent primordiales, car si les porcelets sont lavés, 1, 7 ou 14 jours après leur naissance, seuls ces derniers sont reconnus par leur mère. Bien qu'elles soient perçues par la truie et qu'elles la stimulent pour l'allaitement, les vocalisations émises par les jeunes ne semblent pas lui permettre de discriminer entre des porcelets étrangers et ses propres petits, et ce quel que soit l'âge des animaux.

En revanche, les porcelets sont capables de reconnaître la voix de leur mère dès l'âge de 24-48 heures (Shillito-Walser 1986). Les informations olfactives sont également importantes pour identifier la mère, puisque dès 12 heures après leur naissance, des porcelets sont capables de distinguer l'odeur de la mamelle de leur mère de celle d'une mère étrangère. De même, après 24 heures, ils reconnaissent l'odeur des fèces de leur mère (Horrell et Hodgson 1992).

\section{3 / L'adoption}

Avec la généralisation de la conduite en bandes, l'adoption est devenue une pratique très courante en élevage porcin. Selon J. Dagorn (communication personnelle), pratiquement toutes les portées font l'objet d'échanges de porcelets. Il convient cependant de limiter ou de bien encadrer cette pratique susceptible de favoriser la transmission de la maladie d'amaigrissement du porcelet ou MAP (Madec et al 1999).

L'apparition de lignées de truies hyperprolifiques concourt cependant à une utilisation généralisée des adoptions. 
Lobjectif principal des adoptions dans la période néonatale est d'équilibrer et d'uniformiser les tailles des portées, mais aussi de sauver des porcelets à la suite d'interruption accidentelle ou pathologique de la lactation. Dans la pratique courante, après l'absorption du colostrum, les portées sont homogénéisées, sachant que des porcelets plus petits et plus faibles auront des difficultés à s'imposer à la mamelle s'ils sont en concurrence avec des porcelets plus gros. Pour un génotype tel que le Large White, on laisse un maximum de 12 porcelets (généralement moins chez les primipares) aux truies multipares ayant au moins 12 tétines fonctionnelles.

Il existe différentes techniques d'adoption comportant des variantes propres aux éleveurs, adaptées à leur conduite d'élevage et qui dépendent de critères de faisabilité en terme de disponibilité et d'infrastructures.

Ladoption intra-bande, la plus courante, consiste en un rééquilibrage des tailles des portées et des poids des porcelets après la phase colostrale. Cette technique est parfois utilisée en mettant tous les porcelets de la bande dans le couloir et en constituant les portées en fonction du poids.

Ladoption inter-bandes reste une pratique exceptionnelle et n'est utilisée que pour sauver les porcelets en surnombre dans une bande. L'éleveur fait appel à une truie adoptive de la bande précédente. La truie nourrice est choisie pour ses qualités maternelles et laitières. Ses porcelets sont tous sevrés à 21 jours et sont remplacés par des porcelets âgés de 24 à 48 heures, en surnombre dans leur bande d'origine. Dans la majorité des cas, les porcelets les plus gros sont choisis pour être ainsi adoptés. Une variante consiste à conserver 2 porcelets âgés de 21 jours, pendant 24 heures sous la nourrice. Ceux-ci ont un rôle de démonstrateur et facilitent l'acceptation des nouveau-nés.

Les adoptions multiples permettent une homogénéisation permanente des portées en fonction de la taille des porcelets. Des transferts de porcelets tous les 3 jours permettent des réajustements entre la naissance et 18 jours. Un porcelet peut donc changer jusqu'à 6 fois de portée.

Pratiquée précocement, alors que l'ordre de tétée n'est pas encore établi, l'adoption ne pose pas de problème majeur d'adaptation, ni pour les truies, ni pour les porcelets. Mais elle est parfois pratiquée au-delà d'une semaine d'âge, voire de manière continue jusqu'au sevrage (Straw et al 1998a). L'adoption tardive a l'avantage de mieux uniformiser les poids au sevrage dans une portée et de limiter le retard de croissance des porcelets légers, mais elle est largement contrebalancée par une réduction importante (jusqu'à $20 \%$ ) du poids au sevrage (Straw et al 1998 b). En effet, en dépit de la souplesse relative du lien mère-jeune, les adoptions tardives et répétées génèrent des perturbations profondes dans le comportement des truies et des porcelets (Price et al 1994 ; figure 4). Pratiquée
Figure 4. Incidence de l'adoption sur l'engagement à la tétée des porcelets (d'après Price et al 1994). Observations pendant $6 \mathrm{~h}$ après l'adoption.

Nombre moyen d'engagements à la tétée

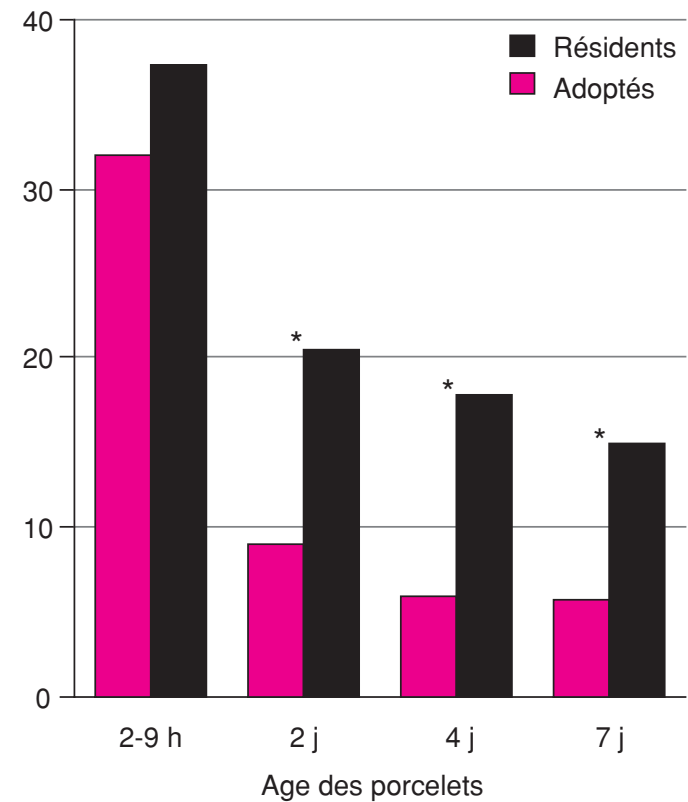

au-delà de 2 jours, l'adoption déstabilise l'ordre à la tétée et désorganise le lien mèrejeune (Horrell et Bennet 1981, Horrell 1982 , Horrell et Hodgson 1986). Au sein de la portée adoptive, les porcelets étrangers passent davantage de temps à errer en émettant des grognements de détresse caractéristiques et montrent une diminution de l'engagement à la tétée. Parallèlement, la truie est perturbée par l'intensité des combats à la mamelle et les cris que poussent les étrangers au moment de la tétée. Cette technique est aujourd'hui largement abandonnée en raison notamment des risques sanitaires (transmission possible de la MAP).

Assez curieusement, une truie en phase de lactation bien établie, séparée de ses propres porcelets, accepte sans grande difficulté une nouvelle portée de 24-36 heures d'âge (Orgeur et al 2000a). Dans ce cas, pendant les 2 à 5 heures qui suivent l'adoption, la truie nourrice est plus agitée, se lève fréquemment et reste davantage couchée sur le ventre que sur le flanc, ne permettant pas l'accès à la mamelle. Mais elle est rarement agressive envers la nouvelle portée. Lorsque les porcelets s'engagent à la tétée, les combats sont du même type que ceux observés dans une portée normale. Au cours de l'intervalle adoption-première tétée, qui correspond à une période de jeûne d'une durée moyenne de 5 heures, les porcelets sont davantage couchés sur le ventre que sur le flanc, ce qui traduit un état d'éveil supérieur (Orgeur et al 2000a). Ils sont également plus regroupés, en contact corporel et sous la source de chaleur additionnelle, suggérant une perturbation de leur thermorégulation. Les vocalisations et l'activité orale envers les congénères sont également accrues pendant cette période (Orgeur et al 2000a). Mesuré à 7 jours, le gain de poids de la portée n'est que légèrement inférieur à celui habituellement observé chez les portées normales (Vourch 1998, Orgeur et al 2000a).

\section{L'adoption est une pratique courante dans les élevages, elle ne pose pas de problème d'adaptation lorsqu'elle est précoce.}


Des porcelets soumis à des adoptions multiples s'agressent beaucoup plus que des porcelets vivant dans des portées non modifiées. La plupart des combats oppose un porcelet résident à un porcelet étranger (80\%) et ils ont pour motif l'accès à une tétine convoitée par eux deux (Robert et Martineau 1998). Dans ces conditions, la truie ne présente pas toujours un réflexe d'éjection de lait lorsqu'elle est sollicitée et qu'elle s'est mise en position d'allaitement. Elle s'assoit ou se lève précipitamment, réduisant ainsi le rythme de tétées. Les truies adoptives passent en moyenne 15 à $30 \%$ de temps en moins couchées sur le flanc que des truies témoins, au jours 4, 7, 13 et 16 post-partum. Le niveau d'agressivité des truies soumises à ces adoptions multiples est également accru et ce comportement est principalement dirigé vers les porcelets étrangers (Robert et Martineau 1998).

\section{4 / Le sevrage}

Le sevrage est une phase naturelle obligatoire du cycle de développement de l'animal. En milieu naturel, il représente l'accoutumance du jeune à l'arrêt de l'allaitement (Counsilman et Lim 1985) et constitue donc une période où le taux d'investissement parental diminue progressivement jusqu'à disparaître (Martin 1984). L'attachement entre la mère et les jeunes, basé en partie sur la satisfaction des besoins nutritionnels des jeunes, s'estompe avec le temps, pour aboutir naturellement à un arrêt de l'allaitement, même si des relations sociales privilégiées subsistent parfois bien au-delà.

En milieu semi-naturel, nous avons vu précédemment que le rythme des tétées et la durée des massages diminuent progressivement au cours de la lactation. Lorsque la laie ou la truie en semi-liberté a la possibilité de se

Figure 5. Evolution de la fréquence des allaitements au cours de la lactation (d'après Boe 1991).

Nombre d'allaitements / $24 \mathrm{~h}$

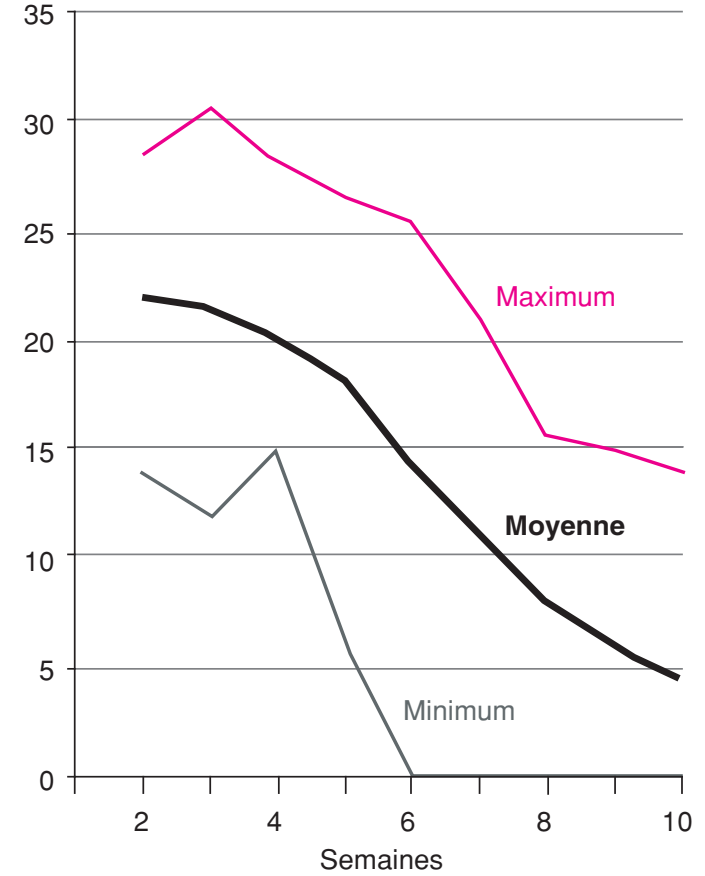

dérober, elle passe moins de temps auprès de sa portée et tend, dès la $4{ }^{\text {ème }}$ semaine post partum, à initier moins de tétées qu'auparavant. A partir de cette période, de plus en plus de tétées se déroulent alors que la truie est en position debout et c'est elle qui les interrompt la plupart du temps. Ces modifications s'accompagnent d'une diminution du massage final de la mamelle par les porcelets, ce qui conduit à une diminution de la quantité de lait produite (Jensen et Recen 1989). Le sevrage survient alors après 10 à 12 semaines d'allaitement selon Boe (1991) (figure 5), après 14 à 17 semaines selon Jensen et Recen (1989).

Dans les conditions d'élevage intensif, c'est l'éleveur qui décide de la séparation soudaine entre la mère et les jeunes. Pour le porcelet, le sevrage représente une situation de stress majeur, puisqu'en plus du changement de logement et d'alimentation qu'il implique, il correspond à la privation de la mère et, le plus souvent, à un changement de groupe social (mélange de portées).

\section{1 / Age au sevrage}

$\mathrm{Au}$ cours de ces 40 dernières années, la réduction de la durée de lactation de 10-12 semaines à 3-4 semaines, a été essentiellement motivée par l'accroissement de la productivité numérique des truies (Dagorn et Aumaître 1979). Le choix de l'âge au sevrage varie en fonction des objectifs et de la capacité technique de l'élevage. Le degré de maturité physiologique des porcelets, qui conditionne l'expression de leur potentiel de croissance (Sève 1986), constitue également un paramètre de choix.

Chez la truie, la période de lactation constitue une période d'anostrus et l'arrêt de la lactation entraîne le déclenchement d'un nouveau cycle de reproduction. Parallèlement, la nécessité de réduire les périodes improductives a justifié la diminution de l'âge au sevrage. Toutefois, la fécondité des truies peut être réduite par des lactations trop courtes (moins de 20 jours) ou trop longues (plus de 35 jours) (Aumaître et al 1976), de sorte que la durée optimale de lactation se situerait, de ce point de vue, entre 22 et 28 jours. Le sevrage à 28 jours permet le maintien d'une fécondité optimale chez la truie (Aumaître et Dagorn 1982, Martinat Botté et al 1984) dont les réserves corporelles sont cependant affectées après 28 jours de lactation. Le nombre de porcelets sevrés par truie et par an est alors d'environ 20, alors qu'il pouvait atteindre 21(Gaubert 1995) et désormais 25 (ITP 2001) avec un sevrage à 18 jours, grâce à l'augmentation du rythme d'utilisation des places de maternité.

Le sevrage à 21 jours, pratiqué dans $15 \%$ environ des élevages français, permet une légère augmentation de la productivité numérique globale des truies, en comparaison d'un sevrage à 28 jours. Il permet aussi de préserver l'état corporel des mères en évitant l'épuisement dû à la $4{ }^{\text {ème }}$ semaine de lactation.

$\mathrm{Au}$ plan national, $80 \%$ des élevages pratiquent un sevrage vers 4 semaines. La limite 
inférieure tolérée selon l'annexe à la directive nouvelle 2001/93/CE est de 4 semaines, sauf dans les élevages conduits en bandes. Un sevrage beaucoup plus précoce peut être toléré, si la non séparation est préjudiciable au bien-être ou à la santé des truies et des porcelets. Un sevrage à une semaine (dit ultraprécoce) peut également être pratiqué pour gérer le surnombre de porcelets dans certaines bandes de truies hyperprolifiques dans lesquelles les capacités d'adoption ont été épuisées (Orgeur et al 2000a).

Une séparation dès la naissance est techniquement possible, dans la mesure où les porcelets sont élevés dans un milieu sans germe et nourris avec un lait artificiel.

Les éleveurs d'Amérique du Nord (Canada, USA), pour des raisons sanitaires, pratiquent fréquemment un sevrage à 14-18 jours, appelé Segregated Early Weaning (SEW) ou Medicated Early Weaning, à un moment où l'immunité systémique est encore élevée. L'éloignement des porcelets d'une source de contamination potentielle que constituent les truies elles-mêmes peut alors être profitable. Toutefois ce type de sevrage n'est pas utilisé en France.

\section{2 / Perturbations du porcelet face au sevrage}

Le sevrage perturbe le porcelet notamment aux plans nutritionnel, thermique, sanitaire et émotionnel qui interfèrent sur son comportement et son équilibre hormonal. Au plan nutritionnel, à une alimentation lactée très hautement digestible succède brutalement une alimentation sèche, non familière et beaucoup moins digestible. Le sevrage est alors associé à une période de sous-consommation d'aliment, de durée variable, au cours de laquelle le porcelet apprend à consommer ce nouvel aliment. En effet, il faut attendre 10-14 jours après le sevrage pour atteindre le niveau de consommation énergétique observé dans les jours qui le précèdent immédiatement. $\mathrm{Au}$ cours de cette période, l'animal puise dans ses réserves adipeuses pour subvenir à ses besoins énergétiques d'entretien et de synthèse protéique, et parfois aux dépenses d'activité physique liées à l'établissement d'un nouvel ordre social lorsque des animaux de différentes portées sont mélangés. L’amaigrissement temporaire qui en résulte accroît la sensibilité thermique de l'animal (Le Dividich et Herpin 1994).

La faible consommation d'aliment immédiatement après le sevrage a aussi des répercussions importantes sur la santé et la fonction digestive du porcelet. Outre qu'elle constitue un facteur de risque de diarrhées (Madec et al 1998), elle se traduit aussi par une réduction importante de la taille des villosités intestinales et par conséquent de la surface de digestion et d'absorption. De même, les activités des enzymes digestives sont temporairement réduites (Le Dividich et al 1998). L'ensemble cumulé pourrait contribuer aux désordres digestifs mentionnés ci-dessus.
Afin d'habituer progressivement les porcelets à consommer et à digérer l'aliment sec proposé au sevrage, de limiter leur déficit nutritionnel au cours de la seconde moitié de l'allaitement et, par conséquent, de rendre moins brutale la transition liée au sevrage, un aliment complémentaire est mis à leur disposition à partir de 10-14 jours d'allaitement. Toutefois cet aliment, distribué dans la très grande majorité des cas sous forme solide, est peu consommé avant 16-18 jours et les quantités ingérées sont très variables, aussi bien entre portées qu'entre porcelets de la même portée (Vantrimponte et Meunier-Salaün 1989 Pajor et al 1991, Fraser et al 1994, Bruininx et al 2001). Une telle variabilité se répercute lors des premiers jours suivant le sevrage. Elle se traduit, pour certains animaux, par des difficultés à ajuster leur ingéré à leurs besoins nutritionnels et peut contribuer à l'apparition de diarrhée et à la perte de poids.

Les aspects comportementaux et hormonaux (glucocorticoïdes : cortisol et catécholamines : adrénaline et noradrénaline) sur lesquels le sevrage influe doivent également être pris en compte pour déterminer les enjeux et les conséquences sur le bien-être et les aspects économiques qui guideront l'éleveur dans le choix de l'âge auquel il sera pratiqué. Malgré les grandes capacités d'adaptation du porcelet, cette séparation soudaine induit des perturbations de son comportement, dont l'intensité est d'autant plus élevée que l'âge au sevrage est plus faible (Worobec et al 1999, Orgeur et al 2000b).

Le sevrage à 21 ou 28 jours ne génère pas de perturbation comportementale majeure et durable chez les porcelets, alors qu'un sevrage à 6-7 jours fait apparaître des changements importants de comportement. Certains de ces changements sont transitoires et étroitement associés à la période de jeûne ( 21 heures en moyenne) et de sous-alimentation qui suit immédiatement le sevrage (vocalisations : figure 6, debout en

Figure 6. Incidence d'un sevrage ultra-précoce (6 jours) sur les vocalisations des porcelets (d'après Orgeur et al 2000a).

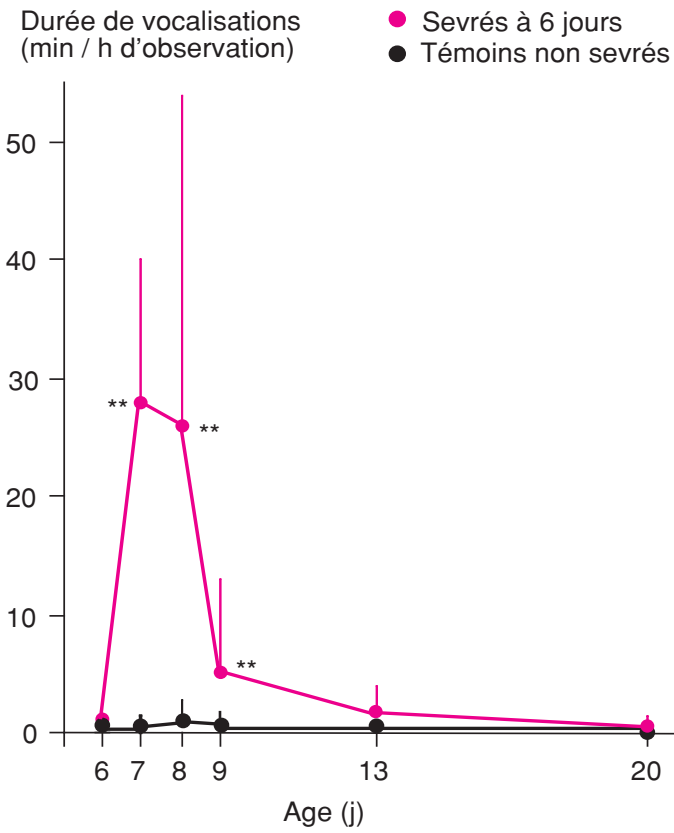


mouvement et en exploration). D'autres perturbations sont plus durables (au moins 2 semaines), en relation avec l'absence de la mère et/ou avec la sous-consommation alimentaire. Elles se traduisent notamment par une grande cohésion de la portée sous la source de chaleur additionnelle et par une augmentation du nombre d'agressions et de massages tétées entre porcelets (Orgeur et al 2000a).

Une élévation du taux urinaire de cortisol, considéré comme indicateur physiologique de stress, est observée dans les deux jours qui suivent un sevrage à 21 ou 28 jours $(\mathrm{V}$. Colson, communication personnelle, Worsaae et Schmidt 1980). Une élévation similaire est notée le lendemain d'un sevrage à 6-7 jours. A l'inverse, les catécholamines urinaires diminuent significativement dans les jours qui suivent la séparation à 6-7 jours, suggérant un déficit énergétique et des problèmes de régulation thermique liés à la période de jeûne (Hay et al 2001).

Après un sevrage à 28 jours, les conséquences négatives en matière de croissance sont limitées aux 2 ou 3 jours qui suivent la séparation.

Les performances globales des porcelets ne sont pas affectées à long terme après un sevrage à 21 jours, bien qu'un retard de croissance d'environ une semaine soit observé (Orgeur et al 2000b). La bonne qualité sanitaire de l'élevage est indispensable, car les porcelets ne commencent qu'alors à développer leur propre immunité.

Avec l'objectif d'améliorer le poids au sevrage des porcelets les plus légers de la portée et d'éviter un amaigrissement trop important de la truie, le sevrage partiel d'une partie des porcelets est parfois pratiqué (entre 18 et 21 jours). En réduisant la compétition à la mamelle, les porcelets restant allaités s'approprient les tétines les plus productives (Sibly et al 1987) et leur croissance s'en trouve améliorée (tableau 3 ; Vesseur et al 1997). Mais l'amélioration n'est que transitoire et en fin de post-sevrage on retrouve les différences de poids entre les porcelets légers et les lourds. Par ailleurs il convient de conserver au moins 6 porcelets par truie pour ne pas risquer un retour en chaleur prématuré (Vesseur et al 1997).

Lintérêt majeur du sevrage ultra-précoce, à l'âge de 5-8 jours, généralement associé à une adoption à 24-48 heures par une truie de la bande précédente, est de permettre la survie des porcelets surnuméraires nés de truies hyperprolifiques. Les conditions sanitaires de l'élevage doivent être irréprochables. Des bâtiments adaptés sont nécessaires, d'abord pour accueillir les porcelets sevrés à 3 semaines, ensuite pour offrir à ceux sevrés à une semaine, des conditions thermiques correspondant à leurs besoins (28 à $\left.30^{\circ} \mathrm{C}\right)$. Dans la majorité des cas, un aliment sec maternisé est distribué, de préférence à un lait artificiel liquide, rapidement dégradé (fermentation) par la température élevée. La croissance des porcelets est affectée pendant environ 2 mois par cette pratique.

Les performances zootechniques traduites en terme de croissance évoluent d'une manière analogue aux perturbations comportementales. Ce sont, en conséquence, les porcelets sevrés le plus précocement qui affichent le déficit de croissance le plus marqué et le plus durable (Worobec et al 1999, Orgeur et al 2000b).

\section{5 / La relation mère-jeunes et le bien-être}

La dernière décennie vient d'être marquée par une préoccupation grandissante du public vis-à-vis de l'animal et de son bien-être. Dans le cadre de l'élevage intensif, les défenseurs de la cause animale se penchent en effet avec de plus en plus d'acuité sur les contraintes imposées à l'animal dit de rente.

$\mathrm{Au}$ fil du temps, après que la domestication de l'animal eut permis à l'homme de l'exploiter pour se nourrir, se vêtir et travailler, il est devenu essentiellement une source de revenus en étant souvent considéré comme un être dénué de sensibilité. C'est au cours des années 1960 que la notion de bien-être a commencé à faire l'objet de débats, puis de textes législatifs visant à protéger l'animal. Celui-ci est désormais considéré comme un être sensible qui doit être placé dans des conditions compatibles avec les impératifs biologiques de son espèce (loi 76-629 du 10 juillet 1976, article 9).

La première des démarches a d'abord eu pour objectif de tenter de définir le bien-être. Selon le rapport Brambell (1965) du Farm Animal Welfare Council (FAWC), cinq impératifs doivent être respectés pour que l'animal soit en situation de bien-être (Dawkins 1983) :

- l'absence de faim, de soif et de malnutrition (aspects physiologiques) ;

- la présence d'abris et le maintien du confort de l'animal (aspects environnementaux)

Tableau 3. Influence d'un sevrage anticipé sur les performances des porcelets (d'après Vesseur et al 1997).

\begin{tabular}{|l|c|c|c|}
\hline & \multicolumn{2}{|c|}{ Porcelets issus de même portée, sevrés à } & Témoins (tous sevrés à \\
& 3 semaines & 4 semaines & 4 semaines) \\
\hline Poids moyen à 3 semaines $(\mathrm{kg})$ & $6,9^{\prime}$ & $5,4^{\mathrm{a}}$ & $6,2^{\mathrm{b}}$ \\
\hline Poids moyen à 4 semaines $(\mathrm{kg})$ & - & $7,3^{\mathrm{a}}$ & $7,7^{\mathrm{b}}$ \\
\hline Gain de poids entre 3 et 4 semaines $(\mathrm{kg})$ & - & $1,9^{\mathrm{a}}$ & $1,5^{\mathrm{b}}$ \\
\hline Poids moyen 10 semaines plus tard $(\mathrm{kg})$ & $21,9^{\mathrm{p}}$ & $23,6^{\mathrm{a}}$ & $24,0^{\mathrm{a}}$ \\
& à 13 semaines & à 14 semaines & à 14 semaines \\
\hline
\end{tabular}

Sur une même ligne, les valeurs suivies de lettres différentes sont significativement différentes à $P<0,05$ (a vs b) ou $\mathrm{P}<0,01$ ( $\mathrm{p} v s \mathrm{q})$. 
- l'absence de maladie ou de blessure (aspect sanitaire) ;

- l'absence de peur ou d'anxiété (aspect psychologique) ;

- la possibilité d'exprimer les comportements normaux de l'espèce (aspects comportementaux).

Les trois premiers points répondent aux exigences des principes classiques de l'élevage, tandis que les deux derniers génèrent plus d'interrogations. Ils posent en effet la question du choix des critères à prendre en compte et de leur objectivité. Le bien-être dont doit bénéficier l'animal est alors un choix de société résultant de la confrontation des exigences éthiques et économiques du consommateur avec ce qu'il peut en coûter à l'animal pour s'adapter aux contraintes imposées (Signoret 1994). D'où l'idée d'une définition complémentaire basée sur une adaptation aux contraintes imposées par l'élevage intensif, celui-ci devant représenter un "coût" acceptable pour l'animal, sans souffrance, ni épuisement (Broom 1987, Signoret 1994). De sorte que l'on entend actuellement par bienêtre, un état physique ou mental en harmonie avec l'environnement (Veissier et al 1999).

La véritable question est de savoir si les contraintes liées à l'élevage actuel sont perçues comme telles par l'animal et comment il est possible d'identifier ces contraintes.

Les critères comportementaux offrent à l'expérimentateur une panoplie de mesures permettant de révéler des situations d'inconfort :

- des comportements anormaux facilement reconnaissables et ayant des conséquences néfastes facilement repérables par l'éleveur (ex : caudophagie chez le porc) ;

- des comportements anormaux facilement décelables, mais sans traduction pathologique évidente (ex : stéréotypies ou activités orales non alimentaires);

- des comportements décelables uniquement lors d'observations systématiques et en comparaison avec un système d'élevage dit de référence.

La difficulté pour l'animal d'exprimer le répertoire comportemental caractéristique de son espèce est considérée comme une source de mal-être. A titre d'exemple, l'impossibilité pour la truie logée en stalle individuelle dépourvue de substrat, de construire un nid au moment de sa parturition pourrait suggérer un état de malêtre, en dépit de ses capacités d'adaptation.

Les critères physiologiques sont centrés sur les hormones considérées comme des indicateurs de stress. Lactivation de l'axe corticotrope (glucocorticoïdes) et de la branche sympathique du système nerveux autonome (catécholamines) est nécessaire pour permettre à l'animal d'améliorer l'efficacité des mécanismes homéostatiques (Dantzer et Mormède 1979). L'exagération de l'effet de ces hormones devient rapidement néfaste, notamment les glucocorticoïdes, en réduisant les défenses immunitaires.

Les critères sanitaires sont souvent considérés par l'éleveur, avec les critères zootech- niques, comme de bons indicateurs du bienêtre de l'animal. Pathologies ou lésions corporelles peuvent en effet être étroitement liées aux conditions d'élevage des animaux (Dantzer 1995). Une production optimale ne permet cependant pas de conclure sur un éventuel état de bien-être chez l'animal, son niveau de performance n'étant pas toujours le reflet de son confort.

Pendant la période mise bas-sevrage, la truie et les porcelets sont soumis, dans le cadre de l'élevage intensif, à diverses contraintes susceptibles d'être des sources de mal-être. Ainsi, les truies en maternité, après avoir subi une restriction de leur registre comportemental (empêchement de la construction d'un nid), sont soumises à une contrainte physique (système de contention) qui limite leur motricité et leur permet difficilement d'interagir avec leurs porcelets ou de se dérober à leurs sollicitations. Dans ces conditions, l'initiation progressive du sevrage, avec l'apprentissage précoce d'une consommation alimentaire sèche est donc fortement restreinte. En revanche, la restriction de mouvements des truies permet de limiter la mortalité des porcelets au cours des premiers jours post-partum. La différence de pertes est évaluée à environ $3 \%$ en faveur du système bloqué, comparé à un système dans lequel la truie peut se retourner (Fraser et al 1997). La liberté de la truie est une des causes de mortalité néonatale rencontrée dans les élevages en plein air, où le taux de perte dans les trois jours suivant la parturition peut atteindre 10 à $20 \%$ du nombre total de porcelets nés. Dans ces élevages, la majorité des pertes est attribuée à des écrasements (Berger 1997, Vieuille et al 1999).

Certaines étapes de la vie de l'animal peuvent être perçues comme des contraintes sociales, quelle que soit la conduite d'élevage. C'est le cas, par exemple, de l'adoption et du sevrage du porcelet, imposés par l'éleveur. Nous avons vu que lors de ces procédures, les critères comportementaux, physiologiques et zootechniques indiquaient une situation de mal-être plus ou moins durable (Orgeur et al 2000a et b), en dépit de la grande souplesse d'adaptation du porc. Les projets visant à imposer une conduite en groupe des truies allaitant, suscitant des conflits liés à la hiérarchie sociale, devront de ce point de vue trouver un compromis pertinent.

Il est important de souligner que l'attention portée par l'éleveur à ses animaux, notamment lors des bouleversements sociaux qu'il leur impose, contribue à limiter les conséquences négatives sur leur santé et leur bienêtre. Il reste que le contexte économique constitue un élément que l'éleveur doit prendre en compte, l'incitant à trouver le meilleur compromis possible en relation avec le respect du bien-être de l'animal et les recommandations et directives récentes.

Des recherches sont déjà ou vont être entreprises dans plusieurs domaines, comme l'amélioration des qualités maternelles des truies hyperprolifiques. Ainsi, le nombre de leurs tétines et leur comportement maternel

Différentes
modalités d'élevage
sont souvent
perçues comme
contraires au bien-
être. Par exemple
la contention des
truies, qui limite
leur mobilité mais
évite l'écrasement
des porcelets, ou
leur logement en
stalle individuelle,
qui les isole du
groupe mais dimi-
nue les interactions
agressives.

\section{Différentes}

sont souvent

perçues comme

contraires au bien-

être. Par exemple

la contention des

truies, qui limite

leur mobilité mais

évite l'écrasement

des porcelets, ou

leur logement en

stalle individuelle,

qui les isole du

nue les interactions

agressives. 
font l'objet d'un programme de sélection par les généticiens. L'une des perturbations les plus notables lors d'une séparation mèrejeunes imposée par l'éleveur à un âge inférieur à celui du sevrage spontané, touche à la transition entre le lait maternel et l'aliment solide. Les études portant sur le mode de présentation et le type d'aliment proposé (liquide versus solide) sont donc nécessaires ; elles sont en cours de réalisation. Il en est de même des combats que les modifications de groupes sociaux génèrent, par exemple lors du mélange de portées au moment du sevrage.

\section{Références}

Algers. B., Jensen P., 1991. Teat stimulation and milk production during early lactation in sows: effects of continuous noise. Can. J. Anim. Sci., 71, 51-60.

Arey D.S., Sancha E.S., 1996. Behaviour and productivity of sows and piglets in a family system and in farrowing crates. Appl. Anim. Behav. Sci., 50, 135-145.

Auldist D.E., King R.H., 1995. Piglet's role in determining milk production in the sow. In : D.P. Hennesy and P.D. Cranwell (eds), Manipulating Pig Production, 114-118. Australasian Pig Science Association, Werribee.

Aumaître A., Dagorn J., 1982. Influence de la durée de lactation sur la fécondité et la prolificité de la truie. Ann. Zootech., 31, 431-444.

Aumaître A., Dagorn J., Legault C., Le Denmat M., 1976. Influence of farm management and breed type on sow's conception-weaning interval and productivity in France. Livest. Prod. Sci., 3, 75-83.

Berger F, 1997. Limiter les pertes sous la mère en élevage de plein air. Résultats de recherche en production porcine. Chambre d'Agriculture Pays de Loire (ed), 9-12.

Beyer M., Jentsch W., 1994. Relationship between milk production of the sow and growth performance in piglets. In : Proceedings of the V International Symposium on Digestive Physiology in Pigs. EAAP Publication, $N^{\circ} 80$, p 220.

Blackshaw J.K., Hagels A.M., 1990. Getting-up and lyingdown behaviours of loose-housed sows and social contacts between sows and piglets during day 1 and day 8 after parturition. Appl. Anim. Behav. Sci., 25, 61-70.

Blackshaw J.K, Blackshaw A.W., Thomas F.J., Newman F.W., 1994. Comparison of behaviour patterns of sows and litters in a farrowing crate and a farrowing pen. Appl. Anim. Behav. Sci., 39, 281-295.

Blecha F., Kelley K.W., 1981. Effects of cold and weaning stressors on the antibody mediated immune response of pigs. J. Anim. Sci., 53, 437-439.

Boe K., 1991. The process of weaning in pigs : when the sow decides. Appl. Anim. Behav. Sci., 30, 47-59.

Boe K., 1993. Maternal behaviour of lactation sows in a loose-housing system. Appl. Anim. Behav. Sci., 35, 327-338.

Broom D.M., 1987. Applications of neurobiological studies to farm animal welfare. In : P.R. Wiepkema \& P.W.M Andrichem (eds), Biology of stress in farm animals, 101110. M. Nijhof Publ., The Hague.

Bruininx E.M.A.M., van der Peet-Schwering C.M.C. Schrama J.W., 2001. Individual feed intake of group housed weaned pigs and health status. In : Varley M.A. and Wiseman J.(eds), The Weaner Pig. Nutrition and Management. CABI Publishing. Wallingford, UK.

Buchenauer D., Dannenmann K., 1979. Research into some factors determining the suckling order in piglets. Berl. Munch. Tierarztl. Wochenschr., 92, 432-437.

Counsilman J.J., Lim L.M., 1985. The definition of weaning. Anim. Behav., 33, 1023-1024.

Dagorn J., Aumaître A, 1979. Sow culling. reasons for and effect on productivity. Livest. Prod. Sci., 6, 167-177.

Dantzer R., 1995. Confort et bien-être des animaux en élevage intensif. Le Point Vétérinaire, 26, 1027-1034.

Dantzer R., Mormède P., 1979. Physiopathologie de la réaction aux agressions. In : Le stress en élevage intensif, 2 , 29-75. Masson, Paris.

Dantzer R., Auffray P., Signoret J.P., 1986. Le comportement. In : J.M. Perez, P. Mornet \& A. Rérat (eds), Le porc et son élevage : bases scientifiques et techniques, 141-156. Maloine, Paris.

Dawkins M.S., 1983. La souffrance animale ou l'étude objective du bien-être animal. Le Point Vétérinaire (Ed), Maisons-Alfort, $152 \mathrm{p}$.
Delcroix I., 1989. Etude du groupe social chez le sanglier (Sus scrofa $L$.) en conditions de semi-liberté : sa fonction adaptative dans la reproduction et l'élevage des jeunes. Thèse Univ. Paris $13,154 \mathrm{p}$.

Deprès E., Caritez J.C., 1991. L'adoption du porcelet aspects comportementaux et zootechniques. INRA, Prod. aspects comportem

Ellendorf F., Poulain D.A., 1984. A means to assess nursing efficiency in the pigs: the study of the milk ejection reflex. Ann. Rech. Vet., 15, 271-274.

Ellendorf F., Forsling M.L., Poulain D.A., 1982. The milk ejection reflex in the pig. J. Physiol., 333, 577-594.

Ely P., Petersen W.E., 1941. Factors involved in ejection of milk. J. Dairy Sci., 24, 211-223.

English P.R., Morrison V., 1984. Causes and prevention of piglet mortality. Pig News Inform., 5, 369-376.

Etienne M., Dourmad J.Y., Noblet J., 1998. The influence of some sow and piglet characteristics and of environmental conditions on milk production. In : Verstegen M.W.A Moughan P.J. and Chrama J.W. (eds), The lactating sow, 285-299. Wageningen Press.

Fraser D., 1973. The nursing and suckling behaviour of pigs. I. The importance of stimulation of the anterior teats. Br. Vet. J., 129, 324-336.

Fraser D., 1980. A review of the behavioural mechanism of milk ejection of the domestic pig. Appl. Anim. Ethol., 6, 247-255.

Fraser D., Feddes J.J.R., Pajor E.A., 1994. The relationship between creep feeding behaviour of piglets and adaptation to weaning: effect of diet quality. Can. J. Anim. Sci., $74,1-6$.

Fraser D., Phillips P.A., Thompson B.K., 1997. Farrowing behaviour and stillbirth in two environments: an evaluation of the restraint-stillbirth hypothesis. Appl. Anim. Behav. Sci., 55, 51-66.

Gaubert P., 1995. Sevrage précoce et réduction des coûts. Porc Magazine, 275, 44-45.

Hartsock T.G., Graves H.B., Baumgardt B.R., 1977. Agonistic behavior and the nursing order in suckling piglets: relationships with survival, growth and body composition. J. Anim. Sci., 44, 320-330.

Hay M., Orgeur P., Lévy F., Le Dividich J., Concordet D. Nowak R., Schaal B., Mormède P, 2001. Neuroendocrine consequences of very early weaning in swine. Physiol. Behav., 72, 263-269.

Hendrix W.F., Kelley K.W., Gaskins C.T., Hinrich D.J., 1978. Porcine neonatal survival and serum gamma globulins. J. Anim. Sci., 47, 1281-1286.

Herpin P., Le Dividich J., 1995. Thermoregulation and energy metabolism. In : Varley M. (ed), The neonatal pig: development and survival, 57-95, IV. CAB International.

Herpin P. Le Dividich J., Hulin J.C., Fillaut M., De Marco F Bertin R, 1996. Effects of the level of asphyxia during delivery on viability at birth and early postnatal vitality of newborn pigs. J. Anim. Sci., 74, 2067-2075.

Horrell I., 1982. Immediate behavioural consequences of fostering 1-week-old piglets. J. Agric. Sci., 99, 329-336.

Horrell I., Bennet J., 1981. Disruption of teat preferences and retardation of growth following cross-fostering of 1 week-old pigs. Anim. Prod., 33, 99-106.

Horrell I., Hodgson J., 1986. The behavioural consequences of fostering in pigs. In : M. Nichelmann (ed) Ethology of domestic animals, 87-92. Privat, I.E.C., Toulouse.

Horrell I., Hodgson J., 1992. The bases of sow-piglet identification. The identification by sows of their piglets and the presence of intruders. Appl. Anim. Behav. Sci., 33, 319-327. 
Hoy S., Lutter C., Wahner M., Puppe B., 1994. Influence of birth weight on the early postnatal vitality of piglets. Deutsche Tierärztliche Wochenschrift, 101, 393-396.

ITP, 2001. Porc Performances 2000. Numéro Spécial : 25 ans de résultats GTTT-GTE-TB. Brochure ITP, 68 p.

Jensen P., 1988. Maternal behaviour and mother-young interactions during lactation in free-ranging domestic pigs. Appl. Anim. Behav. Sci., 20, 297-308.

Jensen P., Recen B., 1989. When to wean. Observations from free-ranging domestic pigs. Appl. Anim. Behav. Sci., 23, 49-60.

Jensen P., Gustafsson M., Augustsson H., 1998. Teat massage after milk ingestion in domestic piglets: an example of honest begging? Anim. Behav., 55, 779-786.

Jones J.E.T., 1966. Observations on the parturition of sow. Br. Vet. J., 122, 420-426.

Klobasa F., Werhahn E., Butler J.E., 1987. Composition of sow milk during lactation. J. Anim. Sci., 64, 1458-1466.

Le Dividich J., 1999. Neonatal and weaner pig: managemennt to reduce variation. In : Cranwell P.D. (ed), Manipulating Pig Production VII, 135-155. Australasian Pig Science Association, Werribee.

Le Dividich J., Herpin P., 1994. Effect of climatic conditions on the performance, metabolism and health status of weaned piglets: a review. Livest. Prod. Sci., 38, 79-90.

Le Dividich J., Noblet J., 1981. Colostrum and thermoregulation in the neonatal pig in relation to environmental temperature. Biol. Neonate, 40, 167-174.

Le Dividich J., Herpin P., Paul E., Strullu F., 1997. Effect of fat content of colostrum on voluntary colostrum intake and fat utilization in newborn pig. J. Anim. Sci., 75, 707-713.

Le Dividich J., Tivey D., Aumaître A., 1998. Gastro-intestinal development and digestive capacity in the young pig. In : Done S., Thomson J. and Varley M. (eds), Proceedings of the 15th IPVS Congress, 299-308. Nottingham University Press, Nottingham, UK.

Léon E., Madec F., 1992. Etude de la phase périnatale chez le porc dans 3 élevages. 2 Santé et performances du porcelet en phase d'allaitement. J. Rech. Porc. en France, 24, 99-108.

Madec F., Bridoux N., Bounaix S., Gestin A., 1998. Measurement of digestive disorders in the piglet at weaning and related risk factors. Prev. Vet. Med., 35, 53-72.

Madec F., Eveno E., Morvan P., Hamon L., Albina E., Truong C., Huet E., Cariolet R., Arnaud C., Gestin A., 1999. Porcine Wasting Disease in France: Description of the disease and impact in affected herds. J. Rech. Porc. en France, 31, 347-354.

Martin P., 1984. The meaning of weaning. Anim. Behav., 32, 1257-1259.

Martinat-Botté F., Badouard B., Terqui M., 1984. Intervalle tarissement-1er oestrus : bilan 1975-1982. Influence de quelques paramètres. J. Rech. Porc. en France, 16, 153-160.

Martineau G.P., 1998. Les troubles de la lactation chez la truie. Atelier porcin. J. Nationales des GTV, Tours

Milon A., Aumaître A., Le Dividich J., Franz J., Metzeger J.J., 1983. Influence of birth prematurity on colostrum composition and subsequent immunity of piglets. Ann. Rech. Vet., 14, 533-540.

Morrow-Tesch J., McGlone J.J., 1990. Sources of maternal odors and the development of odor preferences in baby pigs. J. Anim. Sci., 68, 3563-3571.

Noblet J., Etienne M., 1987. Body composition, metabolic rate and utilization of molk nutrients in suckling piglets. Reprod. Nutr. Dev., 27, 829-839.

Orgeur P., Salaün C., Le Roux T., Venturi E., Le Dividich J., 2000a. Ladoption et le sevrage ultra-précoce : une stratégie pour élever les porcelets en surnombre. J. Rech. Porc. en France, 32, 143-149.

Orgeur P., Colson V., Lemercier H., Le Roux N., Saez E., $2000 \mathrm{~b}$. Le sevrage est-il une source de mal-être pour le porcelet? Coll. Ethol. Appl., Univ. Paris XIII, Villetaneuse, $22-$ celet? Coll. Ethol. Appl.,
24 novembre 2000 , Abst.

Orgeur P., Hay M., Mormède P., Salmon H., Le Dividich J., Nowak R., Schaal B., Lévy F., 2001. Behavioural and immune consequences of early weaning in one week old piglets. Reprod. Nutr. Dev., 41, 321-332.

Pajor E.A., Fraser D., Kramer D.L., 1991. Consumption of solid food by suckling pigs: individual variation and relation to postweaning performance. Appl. Anim. Behav. Sci., 32, 139-155.
Pajor E.A., Weary D.M., Bonenfant M., Fraser D., Kramer D.L. Caceres C., 1997. The effects of housing conditions, piglet diet, and weaning method on sow maternal behaviour and piglet growth. Proc. 31st Int. Cong. of the ISAE, Prague, Abst. n77, p 206.

de Passillé A.M.B., Rushen J., 1989. Suckling and teat disputes by neonatal piglets. Appl. Anim. Behav. Sci., 22, 23-38

de Passillé A.M., Rushen J., Hartsock T.G., 1988. Ontogeny of teat fidelity in pigs and its relation to competition at suckling. Can. J. Anim. Sci., 68, 325-338.

Pedersen L.J., Studnitz M., Jensen K.H., Giersing A.M. 1998. Suckling behaviour of piglets in relation to accessibility to the sow and the presence of foreign litters. Appl Anim. Behav. Sci., 58, 267-279.

Price E.O., Hutson G.D., Price M.I., Borgwardt R., 1994. Fostering in swine as effected by age of offspring. J. Anim. Sci., 72, 1697-1701.

Randall G.C.B., 1972. Observations on parturition in the sow. I. Factors associated with the delivery of the piglets and their subsequent behaviour. Vet. Rec., 90, 178-182.

Robert S., Martineau G.P., 1998. Repeated adoptions during lactation impair the welfare of sows and piglets. Proc. 32nd Int. Cong. of the ISAE, Clermont-Ferrand, Abst. $\mathrm{n}^{\circ} 22, \mathrm{p} 135$.

Rohde-Parfet K.A., Gonyou H.W., 1991. Attraction of newborn piglets to auditory, visual, olfactory and tactil stimuli. J. Anim. Sci., 69, 125-133.

Rooke J., Bland I.M., 2002. The acquisition of passive immunity in the new-born piglet. Livest. Prod. Sci. (in press).

Rossillon-Warnier A., Paquay R., 1984. Development and consequences of teat-order in piglets. Appl. Anim. Behav. Sci., 13, 47-58

Saez E., 2000. L'hyperprolificité et ses conséquences sur la survie, le comportement et la croissance des porcelets. Mémoire DESS, Univ. Paris13, 25 p.

Salmon H., Vaiman M., Schreuer D., 1990. Immunologie du porc. Immunologie comparée, 54, 564-579.

Salmon-Legagneur E., Aumaître A., 1962. Influence de la quantité de lait et de sa composition sur la croissance du porcelet sous la mère. Ann. Zootech., 11, 181-196.

Scheel D.E., Graves H.B., Sherritt G.W., 1977. Nursing order, social dominance and growth in swine. J. Anim. Sci., 45, 219-229.

Schouten W.G.P., 1986. Rearing conditions and behaviour in pigs. Dissertation. Agricultural Univ. of Wageningen, The Netherlands, $155 \mathrm{p}$.

Sève B., 1986. Elevage et sevrage des porcelets. In : J.M. Perez, P. Mornet \& A. Rérat (eds), Le porc et son élevage : bases scientifiques et techniques, 403-429. Maloine, Paris.

Shillito-Walser E., 1986. Recognition of sow's voice by neonatal piglets. Behav., 99, 177-188.

Sibly R.M., Broom D.M., Close W.H., Lindsley J., 1987. Suckling behaviour of piglets half of whose littermates have been removed. Appl. Anim. Behav. Sci., 12, 386 (Abstr.).

Signoret J.P., 1994. Le point de vue d'un chercheur de l'INRA. In : M. Picard, R.H. Porter \& J.P. Signoret (eds) Comportement et bien-être animal, 29-31. Editions INRA, Paris.

Spicer E.M., Driesen S.J., Fahy V.A., Horton B.J., Sim B.J., Jones R.T., Culter R.S., Prime R.W., 1986. Causes of preweaning mortality on a large intensive piggery. Austral. Vet. J., 63, 71-75.

Stolba A., Henderson R., Wechsler B., 1990. The influence of different social and physical environments on the incidence of lactational oestrus in sows. Appl. Anim. Behav. Sci., 27, 269-276.

Straw B.E., Dewey C.E., Bürgi E.J., 1998a. Patterns of crossfostering and piglet mortality on commercial U.S. and Canadian swine farms. Prev. Vet. Med., 33, 83-89.

Straw B.E., Bürgi E.J., Dewey C.E., Duran C.O., 1998b. Effects of extensive crossfostering on performance of pigs on a farm. J. Am. Vet. Med. Assoc., 212, 855-856.

Svendsen J., Bengtsson A.C.H., Svendenson L.S., 1986 Occurrence and causes of traumatic injuries in neonata pigs. Pig News and Information, 7, 159-179.

Thompson B.K., Fraser D., 1988. Variation in piglets weight gains in the first days after birth and their relationship with later performance. Can. J. Anim. Sci., 68, 581-590. 
Vantrimponte M.N, Meunier-Salaün M.C., 1989. Activité alimentaire des porcelets allaités et sevrés en relation avec leur environnement en maternité. J. Rech. Porc. en France, $21,301-308$

Veissier I., Sarignac C., Capdeville J., 1999. Les méthodes d'appréciation du bien-être des animaux d'élevage. INRA Prod. Anim., 12, 113-121.

Vesseur P.C., Kemp B., den Hartog L.A., Noordhuizen J.P.T., 1997. Effect of split-weaning in first and second parity sows on sow and piglet performance. Livest. Prod. Sci. $49,277-285$.

Vieuille C., Berger F., Le Pape G., Bellanger D., 1999. Le comportement maternel de la truie primipare élevée en plein air. Conséquences sur les risques d'écrasement de porcelets sous la mère. Techni-Porc, 22, 13-19.

Vourch S., 1998. Hyperprolificité et bien-être dans l'espè- ce porcine : perturbations comportementales, zootechniques et hormonales liées à l'adoption et au sevrage ultraprécoce. Thèse Vétérinaire, ENV Nantes, 98 p.

Weary D.M., Pajor E.A., Fraser D., Honkanen A.M., 1996. Sow body movements that crushed piglets: a comparison between two types of farrowing accomodation. Appl. Anim. Behav. Sci., 49, 149-158.

Welch A.R., Baxter M.R., 1986. Responses of newborn piglets to thermal and tactile properties of their environment. Appl. Anim. Behav. Sci., 15, 203-215.

Worobec E.K., Duncan I.J.H., Widowski T.M., 1999. Behavioural effects on piglets weaned at 7, 14 and 28 days. Appl. Anim. Behav. Sci., 62, 173-182.

Worsaae H., Schmidt M., 1980. Plasma cortisol and behaviour in early weaned piglets. Acta Vet. Scand., 21, 640-657.

\section{Abstract}

Mother-young relationships in pigs: from birth to weaning.

Modern production methods can induce negative consequences for pig welfare. In wild-pigs, as in domestic pigs reared in natural conditions, parturient females live in a matriarchal group and build a nest to protect their piglets. A selective bond is established between sows and piglets, and suckling continues for a minimum of 10 to 12 weeks. Present rearing conditions have largely modified the life of pigs. Generally, sows are kept in individual pens and can not build a nest. During the suckling period, contacts between the sows and piglets are reduced and only initiated by the young. However, vocal and olfactive exchanges help piglets localise the teats and induce a reciprocal recognition between mother and young during the first 48 hours post partum. The absorption of colostrum as soon as possible after birth is essential for immunity protection and nutrition. The rate of suckling is approximately once per hour during the first weeks of lactation, then it decreases progressively. During the first days post partum, piglets develop a preference for the teats after intensive fights. The attachment between mother and young is not as selective as in ruminating animals. Fostering of piglets is easy when they are less than three days old. Later, adoptive sows accept foreign piglets with difficulty and the young do not attempt to suck for the first hours, or even days. However, the substitution of an entire litter is easily accepted by the sow, even after 3 weeks of nursing. In most cases piglets are weaned at 4 weeks. Weaning before that time is not permitted in intensive breeding, unless health reasons require that the piglets be weaned when they are only one week old. Behavioural and zootechnical disturbances appear correlated with the precocity of weaning. In modern husbandry, the welfare of animals is respected and an attempt is made to avoid anxiety-producing situations and to permit normal behaviour of the species.

ORGEUR P., LE DIVIDICH J., COLSON V., MEUNIER-SALAÜN M.C., 2002. La relation mère-jeune chez les porcins : de la naissance au sevrage. INRA Prod. Anim., 15, 185-198. 